\title{
Large deletions encompassing the TCOF1 and CAMK2A genes are responsible for Treacher Collins syndrome with intellectual disability
}

\author{
Marie Vincent ${ }^{1,7}$, Corinne Collet ${ }^{2,7}$, Alain Verloes ${ }^{3}$, Laetitia Lambert ${ }^{4}$, Christian Herlin ${ }^{5}$, Catherine Blanchet ${ }^{6}$, \\ Elodie Sanchez ${ }^{1}$, Séverine Drunat ${ }^{3}$, Jacqueline Vigneron ${ }^{4}$, Jean-Louis Laplanche ${ }^{2}$, Jacques Puechberty ${ }^{1}$, \\ Pierre Sarda ${ }^{1}$ and David Geneviève ${ }^{\star, 1}$
}

\begin{abstract}
Mandibulofacial dysostosis is part of a clinically and genetically heterogeneous group of disorders of craniofacial development, which lead to malar and mandibular hypoplasia. Treacher Collins syndrome is the major cause of mandibulofacial dysostosis and is due to mutations in the TCOF1 gene. Usually patients with Treacher Collins syndrome do not present with intellectual disability. Recently, the EFTUD2 gene was identified in patients with mandibulofacial dysostosis associated with microcephaly, intellectual disability and esophageal atresia. We report on two patients presenting with mandibulofacial dysostosis characteristic of Treacher Collins syndrome, but associated with unexpected intellectual disability, due to a large deletion encompassing several genes including the TCOF1 gene. We discuss the involvement of the other deleted genes such as CAMK2A or SLC6A7 in the cognitive development delay of the patients reported, and we propose the systematic investigation for $5 \mathrm{q} 32$ deletion when intellectual disability is associated with Treacher Collins syndrome.
\end{abstract}

European Journal of Human Genetics (2014) 22, 52-56; doi:10.1038/ejhg.2013.98; published online 22 May 2013

Keywords: mandibulofacial dysostosis; Treacher Collins syndrome; TCOF1; CAMK2A; intellectual disability

\section{INTRODUCTION}

Mandibulofacial dysostosis (MFD) is defined by abnormal craniofacial development, particularly of the first and second branchial arches. MFD is characterized by malar and mandibular hypoplasia, often associated with cleft palate, conductive hearing loss, and, less frequently, choanal atresia, visceral or skeletal malformations as well as intellectual disability (ID). MFD is not a distinct and specific genetic disease, but is in fact composed of a group of clinically and genetically heterogeneous disorders. Among the several types of MFD, Treacher Collins syndrome (TCS, OMIM 154500) is the most frequent. TCS is also genetically heterogeneous and to date three genes have been implicated in explaining $\sim 80 \%$ of the genetic anomalies in TCS patients (namely TCOF $1,{ }^{1}$ POLR $1 D^{2}$ and $P O L R 1 C^{2}$ ). The remaining genes are still unknown. Heterozygous intragenic deletions/duplications of TCOF1 have been recently described and are a rare cause of TCS. ${ }^{3-4}$ Mutations in EFTUD2 have been identified in patients with MFD and microcephaly ${ }^{5}$ (MFDM, OMIM 610536), characterized by progressive and severe microcephaly, ID and additional malformations, such as choanal and aural atresia, cleft palate, congenital heart defect and esophageal atresia. ${ }^{6}$ However, Gordon et $a l^{6}$ and Luquetti et $a l^{7}$ reported EFTUD2 mutations in patients without microcephaly, suggesting that EFTUD2 could be responsible for MFDM as well as other forms of MFD. 6
We report for the first time two patients with MFD and ID due to the deletion of several genes including TCOF1 and CAMK2A, and discuss the involvement of these genes in the phenotype of the patients.

\section{MATERIALS AND METHODS}

Informed consent was obtained from the parents of the patients for molecular genetic analysis, and publication of the clinical data and pictures.

DNA was extracted from the patient's whole blood sample using the QIAamp DNA Blood Midi Kit (Qiagen, Courtaboeuf, France) according to the supplier's protocol.

The 27 coding exons of TCOF1 and at least $60 \mathrm{bp}$ of intronic sequence flanking the exons were amplified and sequenced with Life Technologies reagents and equipment, see Supplementary data for details.

MLPA analysis was performed using the Salsa MLPA Kit P310 TCOF1 (MRC-Holland, Amsterdam, the Netherlands) and carried out as described by the manufacturer with capillary electrophoresis on an ABI 3130 DNA Analyzer (Life Technologies, Courtaboeuf, France), GeneMapper software version 4.0 (Life Technologies) and MRC Coffalyzer MLPA-Dat Software (MRC-Holland). Additional information about commercially available probe sets is available at http://www.mrc-holland.com.

CNV detection was performed using array-CGH Agilent (Agilent Technologies, Les Ulis, France) 180k for patient 1 and array-CGH Agilent 244k for patient 2. Experiments followed standard and manufacturer's recommendations. DNA sequence information refers to the public UCSC database NCBI37 (Hg19). Genome assemblies were NCBI136/hg18 UCSC for patient 1 and GRCh37/hg19 for patient 2.

\footnotetext{
${ }^{1}$ Département de Génétique Médicale, CHRU Montpellier, Faculté de Médecine de Montpellier-Mimes, Université Montpellier 1, Montpellier, France; ${ }^{2}$ Service de Biologie Moléculaire, Hôpital Lariboisière, Paris, France; ${ }^{3}$ Département de Génétique Médicale, Hôpital Robert Debré, Paris, France, Université Denis Diderot Sorbonne-Paris, INSERM U676, Département de Génétique, Sart Tilman University Hospital, Liège, Belgium; ${ }^{4}$ Service de Génétique Médicale, CHRU de Nancy-Brabois, Nancy, France; ${ }^{5}$ Service de Chirurgie Plastique Infantile, CHRU Montpellier, Montpellier, France; ${ }^{6}$ Service d'Oto-rhino-laryngologie, CHRU Montpellier, Montpellier, France

${ }^{7}$ These authors contributed equally to this work.

*Correspondence: Professor D Geneviève, Departement de Génétique, CHU Montpellier, Hopital Arnaud de Villeneuve, 371, avenue du Doyen Gaston Giraud, 34295 Montpellier cedex 5, France. Tel: + 334 67336564; Fax: + 334 67336052; E-mail: d-genevieve@chu-montpellier.fr
}

Received 3 December 2012; revised 8 April 2013; accepted 10 April 2013; published online 22 May 2013 


\section{RESULTS}

\section{Clinical reports}

Patient 1. She was born at term after a normal pregnancy, to unrelated and unaffected Belgian parents. Birth weight was normal $(3100 \mathrm{~g},-0.5 \mathrm{SD})$. Other parameters were unknown. Family history was uneventful. Diagnosis of MFD was made at birth based on malar hypoplasia, down-slanted palpebral fissures, microtia and microretrognathia. The baby had bilateral conductive hearing loss ( $-60 \mathrm{~dB}$ on the right side and $-70 \mathrm{~dB}$ on the left side), which was treated with bone-anchored hearing aid implants. She walked at 18 months and spoke with a delay initially attributed to hearing loss. However, she had persistent learning difficulties despite the hearing aid. She was given special schooling and acquired the basics of reading and writing, but had attention deficit, major difficulties in logicalmathematical skills and persistent clumsiness. She also had difficulties in social interaction, but did not present objective autistic features. At the last evaluation at the age of 14 years, growth parameters and head circumference were normal (weight: $55.5 \mathrm{~kg}$, mean (M); height: $160 \mathrm{~cm}, \mathrm{M}$; and OFC: $55.5 \mathrm{~cm}, \mathrm{M})$. Her facial features fit the diagnosis of MFD, namely down-slanted palpebral fissures with mild colobomatous cleft of the lower lid, small mandible with class III malocclusion and low-set and dysplastic ears (Figure 1a). The voice was high pitched and hypernasal.

Patient 2. He was born at term after a pregnancy marked by maternal smoking (10 cigarettes per day). BW was $2800 \mathrm{~g}(-2 \mathrm{SD})$, $\mathrm{BL}$ was $50 \mathrm{~cm}(\mathrm{M})$ and $\mathrm{BOFC}$ was $35 \mathrm{~cm}(\mathrm{M})$. The low weight was attributed to smoking during pregnancy and normalized quickly. Parents were unrelated and unaffected, of French origin with an uneventful family history. TCS was diagnosed at 6 days of life based on facial features. The neonatal period was marked by feeding difficulties due to craniofacial malformation and required nasogastric tube. Newborn neurological examination was normal. The baby had bilateral moderate conductive hearing loss, which was treated with auditory prostheses. He spoke no words at the age of 26 months, and only 3 words at 3 years. The speech delay was initially thought to be due to neglect to wear the hearing aids, but he thereafter developed additional learning difficulties. The patient had a neuro-psychological evaluation at 8.5 years. Performance IQ was at 71, working memory was at 58 . He could neither read nor write. He presented behavioral disorders, violence and aggressiveness, and required treatment with risperidone. He attended special classes in an institute for patients with hearing impairment. At the last evaluation (Figure 1b) at age 9 years and 11 months, growth, weight and head circumference were in the normal range $(135 \mathrm{~cm}, 30 \mathrm{~kg}$ and $54.5 \mathrm{~cm})$. He presented with facial features of MFD, namely down-slanted palpebral fissures, malar hypoplasia, micrognathia and microtia, and mild ID.

Molecular study. Direct sequencing of TCOF1 was negative in both patients. MLPA of TCOF1 showed a complete deletion of the gene in the two patients (Figures $2 \mathrm{a}$ and $2 \mathrm{~b}$ ). For patient 1, MLPA of both parents showed no deletion of TCOF1. For patient 2, only the DNA of the mother was available and MLPA of TCOF1 was normal. CGH array in patient 1 showed a deletion of $1 \mathrm{Mb}$, encompassing TCOF1,
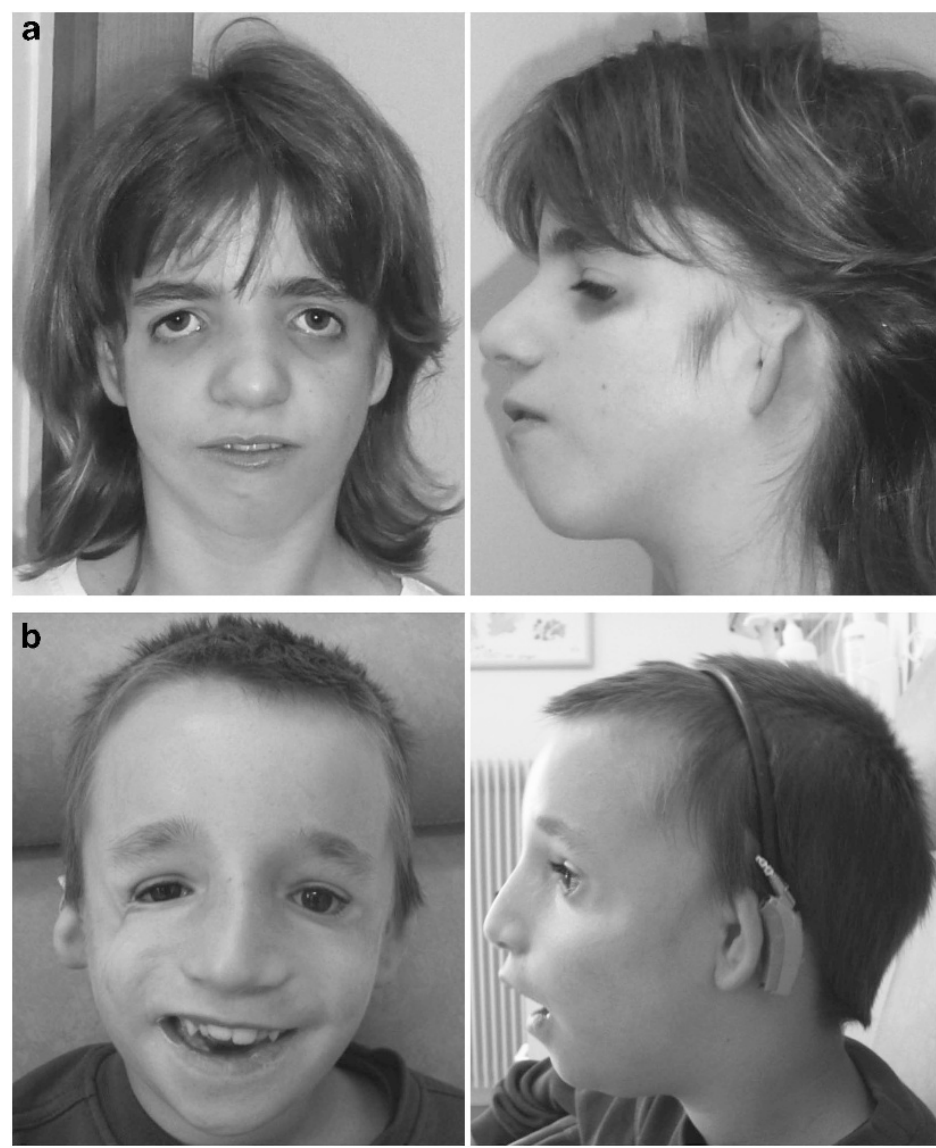

Figure 1 (a) Front and lateral view of patient 1, at the age of 14 years. Note down-slanted palpebral fissures, coloboma of the lower lid, malar hypoplasia and micrognathia. (b) Front and lateral view of patient 2, at the age of 9 years. Note down-slanted palpebral fissures, malar hypoplasia, micrognathia and microtia with audioprosthesis. 
a Patient 1

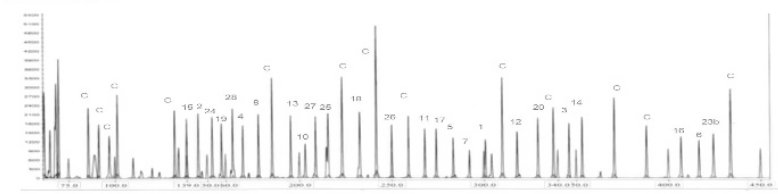

Control

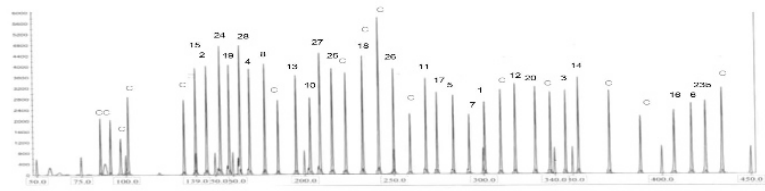

C

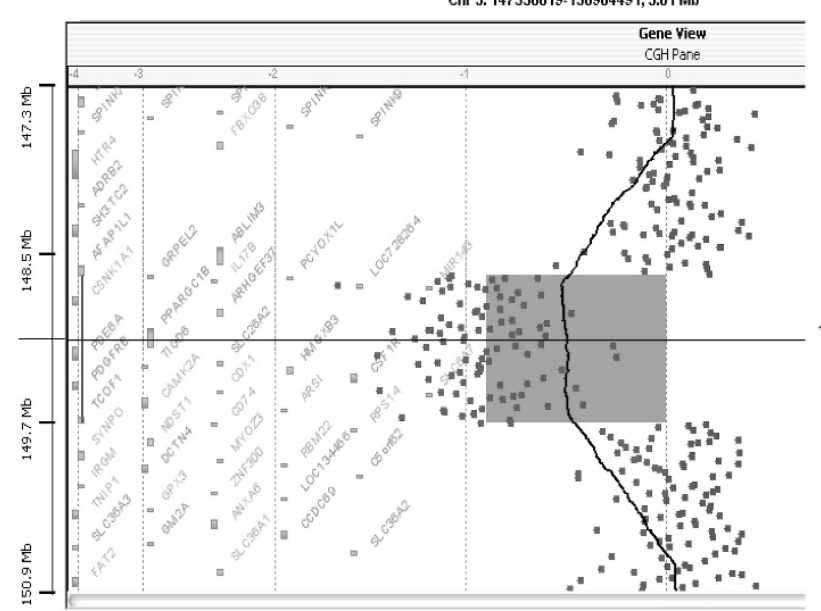

Patient 1 b Patient 2

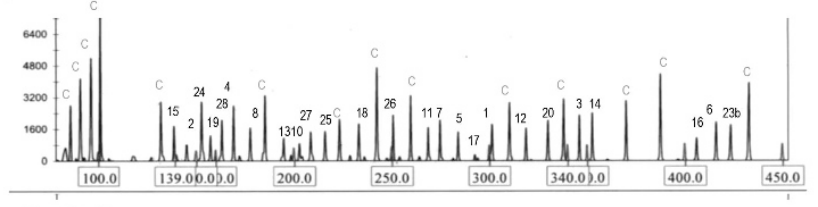

Control

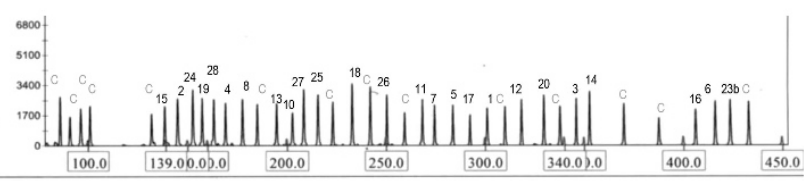

d

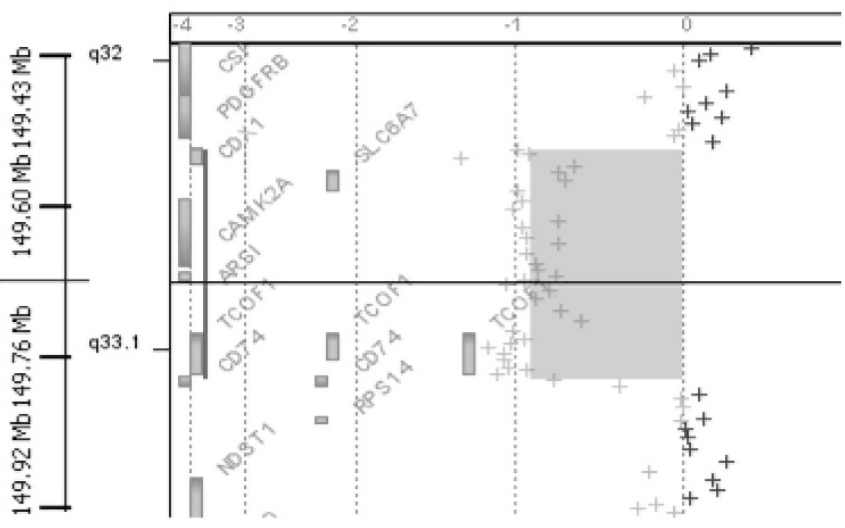

Patient 2

Figure 2 (a) MLPA analysis of patient 1, showing a half-dose for all exons of TCOF1, revealing a total deletion of the gene, c correspond to internal controls. (b) MLPA analysis of patient 2, showing a half-dose for all exons of TCOF1, revealing also a total deletion of the gene. (c) CGH array of patient 1 , revealing a deletion of $1 \mathrm{Mb}$ encompassing TCOF1, GRPEL2, PCYOX1L, IL17B, CSNK1A1, FLJ41603, PPARGC1B, PDE6A, SLC26A2, TIGD6, CSF1R, PDGFRB, CDX1, SLC6A7, CAMK2A, and ARSI. (d) CGH array of patient 2, revealing a deletion of 262000 bp encompassing TCOF1, CDX1, SLC6A7, $C A M K 2 A, A R S I$ and $C D 74$.

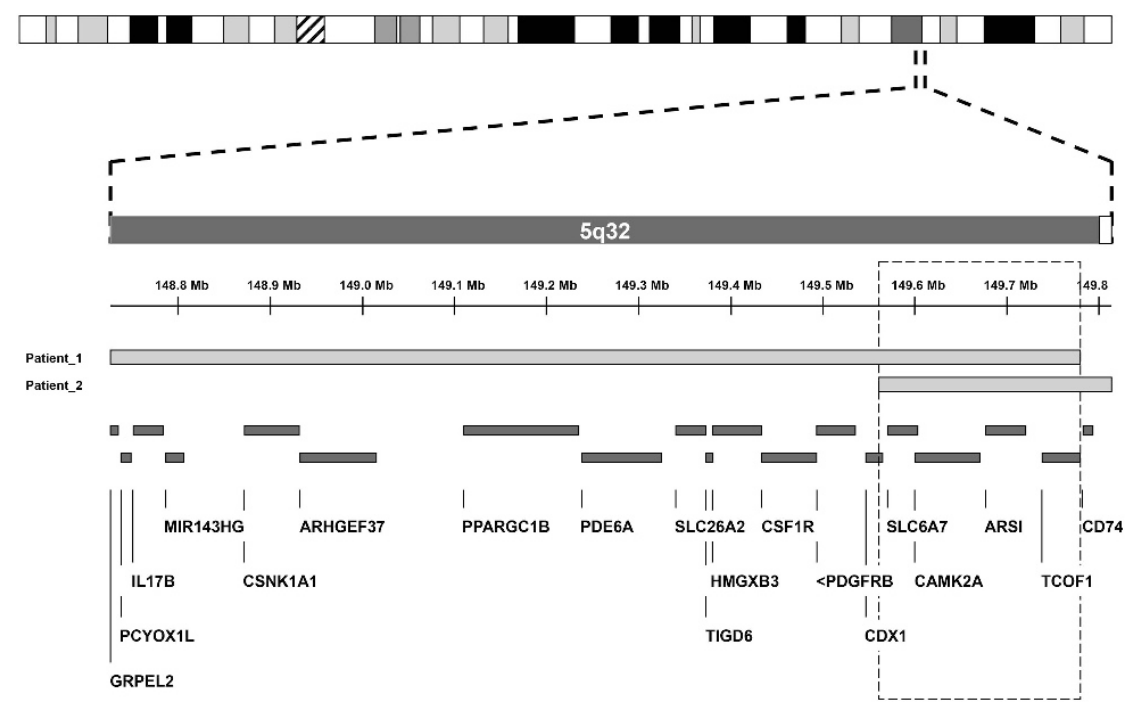

Figure 3 Schematic representation of the $5 q 32$ deletions with their content of genes by the patients reported.

GRPEL2, PCYOX1L, IL17B, CSNK1A1, FLJ41603, PPARGC1B, PDE6A, SLC26A2, TIGD6, CSF1R, PDGFRB, CDX1, SLC6A7, $C A M K 2 A$, and ARSI (Figure 2c). CGH array in patient 2 showed a deletion of $262 \mathrm{~kb}$, encompassing TCOF1, CDX1, SLC6A7, CAMK2A, $A R S I$ and CD74 (Figure 2d). The common deleted genes in the two patients are TCOF1, CAMK2A, CDX1, ARSI and SLC6A7 (Figure 3). 
No patient with similar deletion was reported in the Decipher Database.

\section{DISCUSSION}

MFD is a clinically and genetically heterogeneous group of disorders associated with craniofacial features, visceral and/or skeletal malformations and sometimes ID. The clinician's orientation towards the diagnosis of a specific kind of MFD is based on different combinations of these clinical features. Usually the observation of ID in a patient with MFD encourages the clinician to consider a diagnosis other than TCS. Among the etiologies of MFD with ID, the MFDM or MFD Guion-Almeida type was recently linked to mutations in EFTUD2. ${ }^{5-7}$ Here, we report on two patients with MFD, mild ID and normal OFC, sharing a deletion of TCOF1, CAMK2A, CDX1, $A R S I$ and SLC6A7. In an attempt to determine which genes (or combination of genes) are responsible for the phenotype observed in the two patients, we considered the known functions of the genes located in the minimal critical region.

TCOF1 encodes for a protein called treacle, which is part of a complex implicated in ribosomal RNA biogenesis and is involved in the proliferation and differentiation of neural crest cells in the first and second branchial arches during early embryogenesis. ${ }^{8}$ To our knowledge, mutations in TCOF1 are responsible for the facial features observed in TCS, but have never been reported to cause ID. However, it has been shown recently that Tcofl \pm mice exhibit reduced brain size as a consequence of defects in neural progenitor maintenance. ${ }^{9}$ We believe that deletion of TCOF1 is responsible for the facial features observed in the two patients we reported on. We also believe that the role of TCOF1 in ID observed in the two reported patients is unlikely. Indeed, even if Tcofl is involved in the control of brain size in mice, patients with haploinsufficiency of TCOF1 by point mutation or intragenic deletion/duplication usually do not present with ID or microcephaly. However, we cannot rule out a combinatorial effect of the deletion of TCOF1 with the other deleted genes.

The CAMK2A gene (OMIM 114078) encodes the alpha subunit of calcium/calmodulin-dependent protein kinase II (CaM kinase II), which is present in abundance in the brain where it constitutes a major constituent of the postsynaptic density. ${ }^{10}$ Autophosphorylation of CAMK2A after NMDA receptor activation seems to be essential for contextual long-term memory formation. ${ }^{11}$ Different models have shown that CAMK2A is necessary for hippocampal place cell stability ex vivo and for spatial learning, long-term memory, decreased fear response and increase in defensive aggression in vivo. ${ }^{12}$ CAMK2A may modulate the synaptic events required for the consolidation of memory traces in cortical networks and may have a critical role in plasticity and learning. ${ }^{10}$ Mice heterozygous for a null mutation of Camk2a have profoundly dysregulated behaviors and impaired neuronal development in the dentate gyrus. ${ }^{13}$ No heterozygous variant predicting to lead to haploinsufficiency in CAMK2A has been identified in Exome Variant Server (EVS; http://evs.gs. washington.edu). Integrating all these data, we suggest that CAMK2A could be responsible for the ID observed in the two reported patients.

The SLC6A7 gene (OMIM 606205) encodes a neurotransmitter L-Proline transporter and is a member of the $\gamma$-aminobutyric acid (GABA) neurotransmitter gene family. ${ }^{14}$ It is expressed mostly in the hypothalamus and the hippocampus. ${ }^{15}$ To date, this gene has been only described as a susceptibility gene for asthma. ${ }^{14}$ Based on the known function of this gene (gene belonging to the GABA neurotransmitter family and expressed in the brain), we can only speculate that this gene could participate to the ID observed in the two reported patients. However, two frameshift variants (with unknown modification of function using polyphen web resource) have been observed in EVS.

The caudal-type homeobox transcription factor 1 gene (CDX1, OMIM 600746) is known to be required for anterior-posterior regional identity in Drosophila. In humans, it is an essential transcription factor for intestinal differentiation, and may contribute to intestinal metaplasia in the stomach. ${ }^{16}$ No digestive problems were observed in the patients and only one nonsense variant with unknown modification of function using polyphen web resource was observed in EVS. We suggest that this gene is not involved in the phenotype of the two reported patients.

The ARSI gene (OMIM 610009) encodes for arylsulfatase I, which catalyzes the hydrolysis of sulfate esters and is involved in hormone biosynthesis, modulation of cell signaling, and degrading of macromolecules. ${ }^{17}$ The pattern of expression of the ARSI gene is mainly restricted to embryonic tissue and some cancer cell lines, ${ }^{18}$ suggesting that ARSI is also not involved in the phenotype of the patients. In addition, no heterozygous variant predicting to lead to haploinsufficiency was observed in EVS for ARSI.

In conclusion, we describe for the first time, two patients with MFD and ID and for whom a deletion encompassing TCOF1 and $C A M K 2 A$ has been identified. Based on the knowledge of the deleted genes in the two patients, we hypothesize that CAMK2A is in part responsible for ID and TCOF1 is responsible for the facial features. However, we cannot rule out a combinatorial effect with the other deleted genes. To our opinion, the genetic disorder observed in the two patients is to be considered as a contiguous gene deletion syndrome due to the combination of the effect of the loss of the TCOF1 gene and other genes such as CAMK2A. We suggest investigating for $5 \mathrm{q} 32$ deletion covering TCOF1 and CAMK2A if TCS is associated with ID without microcephaly, and to analyze EFTUD2 if TCS is associated with ID and microcephaly.

\section{CONFLICT OF INTEREST}

The authors declare no conflict of interest.

\section{ACKNOWLEDGEMENTS}

We thank the patients and the family members for their support. Part of this work was supported by the French Franceschetti-Treacher Collins association Coline, by the research program 'Programme Hospitalier de Recherche Clinique Régional' Languedoc-Roussillon and by the Direction Générale de l'Organisation des Soins (DGOS).

1 The Treacher Collins Syndrome Collaborative Group: Positional cloning of a gene involved in the pathogenesis of Treacher Collins syndrome. Nat Genet 1996; 12: 130-136.

2 Dauwerse JG, Dixon J, Seland S et al: Mutations in genes encoding subunits of RNA polymerases I and III cause Treacher Collins syndrome. Nat Genet 2011; 43: 20-22.

3 Beygo J, Buiting K, Seland S et al: First report of a single exon deletion in TCOF1 causing Treacher Collins syndrome. Mol Syndromol 2012; 2: 53-59.

4 Bowman M, Oldridge M, Archer $\mathrm{C}$ et al: Gross deletions in TCOF1 are a cause of Treacher-Collins-Franceschetti syndrome. Eur J Hum Genet 2012; 20: 769-777.

5 Lines MA, Huang L, Schwartzentruber J et al: Haploinsufficiency of a spliceosomal GTPase encoded by EFTUD2 causes mandibulofacial dysostosis with microcephaly. Am J Hum Genet 2012; 90: 369-377.

6 Gordon CT, Petit F, Oufadem M et al: EFTUD2 haploinsufficiency leads to syndromic oesophageal atresia. J Med Genet 2012; 49: 737-746.

7 Luquetti DV, Hing AV, Rieder MJ et al: 'Mandibulofacial dysostosis with microcephaly' caused by EFTUD2 mutations: expanding the phenotype. Am J Med Genet A 2013; 161: 108-113.

8 Dixon J, Jones NC, Sandell LL et al: Tcof1/Treacle is required for neural crest cell formation and proliferation deficiencies that cause craniofacial abnormalities. Proc Natl Acad Sci USA 2006; 103: 13403-13408. 
9 Sakai D, Dixon J, Dixon MJ, Trainor PA: Mammalian neurogenesis requires TreaclePlk1 for precise control of spindle orientation, mitotic progression, and maintenance of neural progenitor cells. PLoS Genet 2012; 8: e1002566.

10 Elgersma Y, Fedorov NB, Ikonen S et al: Inhibitory autophosphorylation of CaMKII controls PSD association, plasticity, and learning. Neuron 2002; 36 493-505.

11 Irvine EE, Danhiez A, Radwanska $\mathrm{K}$ et al: Properties of contextual memory formed in the absence of $\alpha$ CaMKII autophosphorylation. Mol Brain 2011; 4: 8 .

12 Chen S, Xu Y, Xu B et al: CaMKII is involved in cadmium activation of MAPK and mTOR pathways leading to neuronal cell death. J Neurochem 2011; 119: 1108-1118.

13 Yamasaki N, Maekawa M, Kobayashi K et al: Alpha-CaMKII deficiency causes immature dentate gyrus, a novel candidate endophenotype of psychiatric disorders. Mol Brain 2008; 1: 6
$14 \mathrm{Kim} \mathrm{J}-\mathrm{H}$, Cheong HS, Park B-L et al: A new association between polymorphisms of the SLC6A7 gene in the chromosome 5q31-32 region and asthma. J Hum Genet 2010; 55: 358-365.

15 Shafqat S, Velaz-Faircloth M, Henzi VA et al: Human brain-specific L-proline transporter: molecular cloning, functional expression, and chromosomal localization of the gene in human and mouse genomes. Mol Pharmacol 1995; 48: 219-229.

16 Rau TT, Rogler A, Frischauf $M$ et al: Methylation-dependent activation of CDX1 through NF-kB: a link from inflammation to intestinal metaplasia in the human stomach. Am J Pathol 2012; 181: 487-498.

17 Sardiello M, Annunziata I, Roma G, Ballabio A: Sulfatases and sulfatase modifying factors: an exclusive and promiscuous relationship. Hum Mol Genet 2005; 14: 3203-3217.

18 Obaya AJ: Molecular cloning and initial characterization of three novel human sulfatases. Gene 2006; 372: 110-117.

Supplementary Information accompanies this paper on European Journal of Human Genetics website (http://www.nature.com/ejhg) 